University of New Hampshire

University of New Hampshire Scholars' Repository

Space Science Center

Institute for the Study of Earth, Oceans, and

Space (EOS)

2000

\title{
Improved COMPTEL maps of the milky way
}

\author{
H Bloemen \\ Space Research Organization of the Netherlands \\ K Bennett \\ ESTEC \\ W Collmar \\ Max-Planck-Institut für extraterrestriche Physik \\ R Diehl \\ Max-Planck-Institut für extraterrestriche \\ W Hermsen \\ Space Research Organization of the Netherlands (SRON)
}

See next page for additional authors

Follow this and additional works at: https://scholars.unh.edu/ssc

Part of the Astrophysics and Astronomy Commons

\section{Recommended Citation}

Improved COMPTEL maps of the milky way Bloemen, $\mathrm{H}$. and Bennett, $\mathrm{K}$. and Collmar, W. and Diehl, R. and Hermsen, W. and lyudin, A. and Knödlseder, J. and McConnell, M. and Ryan, J. and Schönfelder, V. and Strong, A., AIP Conference Proceedings, 510, 586-590 (2000), DOI:http://dx.doi.org/10.1063/1.1303270

This Conference Proceeding is brought to you for free and open access by the Institute for the Study of Earth, Oceans, and Space (EOS) at University of New Hampshire Scholars' Repository. It has been accepted for inclusion in Space Science Center by an authorized administrator of University of New Hampshire Scholars' Repository. For more information, please contact Scholarly.Communication@unh.edu. 


\section{Authors}

H Bloemen, K Bennett, W Collmar, R Diehl, W Hermsen, A lyudin, J Knodlseder, Mark L. McConnell, James M. Ryan, V Schonfelder, and A W. Strong 


\section{AIP $\mid$ Proceedings}

\section{Improved COMPTEL maps of the milky way}

H. Bloemen, K. Bennett, W. Collmar, R. Diehl, W. Hermsen, A. Iyudin, J. Knödlseder, M. McConnell, J. Ryan, V.

Schönfelder, and A. Strong

Citation: AIP Conference Proceedings 510, 586 (2000); doi: 10.1063/1.1303270

View online: http://dx.doi.org/10.1063/1.1303270

View Table of Contents: http://scitation.aip.org/content/aip/proceeding/aipcp/510?ver=pdfcov

Published by the AIP Publishing

\section{Articles you may be interested in}

EGRET (GeV) Blazars

AIP Conf. Proc. 558, 324 (2001); 10.1063/1.1370801

Galactic diffuse gamma ray emission $>10 \mathrm{GeV}$

AIP Conf. Proc. 558, 171 (2001); 10.1063/1.1370789

The diffusive galactic GeV/TeV ramma-ray background: Sources vs. transport

AIP Conf. Proc. 515, 281 (2000); 10.1063/1.1291380

The cosmic diffuse gamma-ray background measured with COMPTEL

AIP Conf. Proc. 510, 467 (2000); 10.1063/1.1307028

Detecting the attenuation of blazar gamma-ray emission by extragalactic background light with GLAST

AIP Conf. Proc. 510, 759 (2000); 10.1063/1.1303301 


\title{
Improved COMPTEL maps of the Milky Way
}

\author{
H. Bloemen*, K. Bennett"l, W. Collmar ${ }^{\dagger}$, R. Diehl ${ }^{\dagger}$, W. Hermsen*, \\ A. Iyudin ${ }^{\dagger}$, J. Knödlseder ${ }^{\S}$, M. McConnell ${ }^{\ddagger}$, J. Ryan $^{\ddagger}$, \\ V. Schönfelder ${ }^{\dagger}$, A. Strong ${ }^{\dagger}$ \\ *SRON-Utrecht, Sorbonnelaan 2, 3584 CA Utrecht, The Netherlands \\ † MPE, Postfach 1603, 85740 Garching, Germany \\ ${ }^{*}$ Space Science Center, UNH, Durham NH 03284, USA \\ ${ }$ Astrophysics Division, ESTEC,2200 AG Noordwijk, The Netherlands \\ $\S$ CESR, CNRS/UPS, BP 4346, 31028 Toulouse Cedex, France
}

\begin{abstract}
In the course of the mission we have gradually developed an analysis method that separates in an iterative manner the celestial emission and the (a priori unknown) instrumental background. It has become our standard analysis tool for point sources. We illustrate here that this method is widely applicable now. It provides mutually consistent sets of model-fitting parameters (spectra) and sky maps, both for continuum and line studies. Because of the wide applicability, it has been possible to make various cross-checks while building up confidence in this procedure.
\end{abstract}

\section{INTRODUCTION}

COMPTEL has the capability of mapping the sky in the $1-30 \mathrm{MeV}$ regime with an angular resolution of $1^{\circ}-3^{\circ}$ and an energy resolution of 5-10\% FWHM [21]. The data analysis, however, is certainly not trivial. It is complicated by the dominant instrumental background and by the complex response of the instrument. The background exceeds the celestial (galactic) signal by a factor of roughly 50 and it has significantly changed during the mission. In mapping $\gamma$-ray line emission, the removal of celestial continuum radiation introduces an additional challenging problem. In order to study point sources along the Milky Way (apart from pulsars), the diffuse emission has to be accounted for carefully. We have gradually learned how to deal more accurately with this variety of important 'background' aspects.

The COMPTEL imaging data space is 3-dimensional, consisting of two spatial coordinates (the scatter direction $\chi, \psi$ ) and the Compton scatter angle $\bar{\varphi}[21]$. A sufficiently accurate independent estimate of the instrumental background in this data space is not available. We have therefore developed a filtering technique, of which the basic principle is described in [2]. This has become our standard method for point-source studies (through forward folding in maximum-likelihood analyses). It is based on the fact that the $(\chi, \psi)$ structure of the data cube is to first order

CP510, The Fifth Compton Symposium, edited by M. L. McConnell and J. M. Ryan (1) 2000 American Institute of Physics 1-56396-932-7/00/\$17.00 
well described by known geometry characteristics, with deviations being largely independent of $\bar{\varphi}$. In order to enable accurate quantitative studies of extended emission (or to account for its presence in source studies), an iterative approach was added to this method, with background modeling and model fitting being performed simultaneously. Also, all viewing periods (VPs) are handled separately now to account accurately for changes in the background during the mission [10].

\section{CONTINUUM EMISSION}

Our prime goal here is to derive maps that can be used in a quantitative manner. Previous sky-mapping attempts have been presented by Strong et al. [22,25] and by Bloemen et al. [1,4], the latter [4] showing first results from the current approach. In all analyses addressed in this paper, our first step is the instrumentalbackground modeling and simultaneous (all-sky) model fitting mentioned above. The purpose of the model fitting is to estimate (and account for) the total sky flux in the map as well as its global distribution while generating the background model. Previously, background scaling factors had to be chosen and emission near intense sources was suppressed [25]. The resulting background model is then used to generate the actual map, for which we use here maximum-entropy imaging. In an iterative manner, through likelihood optimization, background models are first determined for each individual viewing period, while model intensity distributions are fitted simultaneously to the combined set of observations. For approximation of the expected continuum emission, we used a total gas column density map as a tracer of the bremsstrahlung emission (from HI and CO data), an inverse-Compton model [27], an isotropic component, and the strongest sources, Crab and Cyg X-1. Although the sky models used here provide a good global description of the data, contributions from ensembles of sources with similar large-scale distributions can of course not be excluded. But this is in principle irrelevant for the background modeling and subsequent imaging. We have verified that using other model intensity distributions (e.g. various combinations of 2 to 3 rather arbitrarily chosen smooth components) gives very similar results, provided, of course, that these models indeed enable a global description of the $\gamma$-ray Milky Way.

The model-fitting result is interesting in itself (spectra, hypothesis testing), but is not addressed here in further detail. The results of such large-scale model fitting procedures have already turned out to be strongly influenced by different handlings of the instrumental background (c.f. Strong et al. [22,23,26] \& Bloemen et al. [3,4]).

Fig. 1a shows the 1-30 MeV map that we have extracted with this technique. Fig. 1b shows the corresponding map of candidate sources (see also [7]), which was obtained by adding the fitted sky models to the instrumental-background model. These maps are of the ' $\nu F_{\nu}$ ' type; they are sums of 3 maps $(1-3,3-10$, and 10-30 $\mathrm{MeV}$ ), which we have added together with weight factors such that all 3 contribute equally under the assumption of an $E^{-2}$ photon spectrum (so characteristics of each band are visible in these images). The significance of small-scale features has 


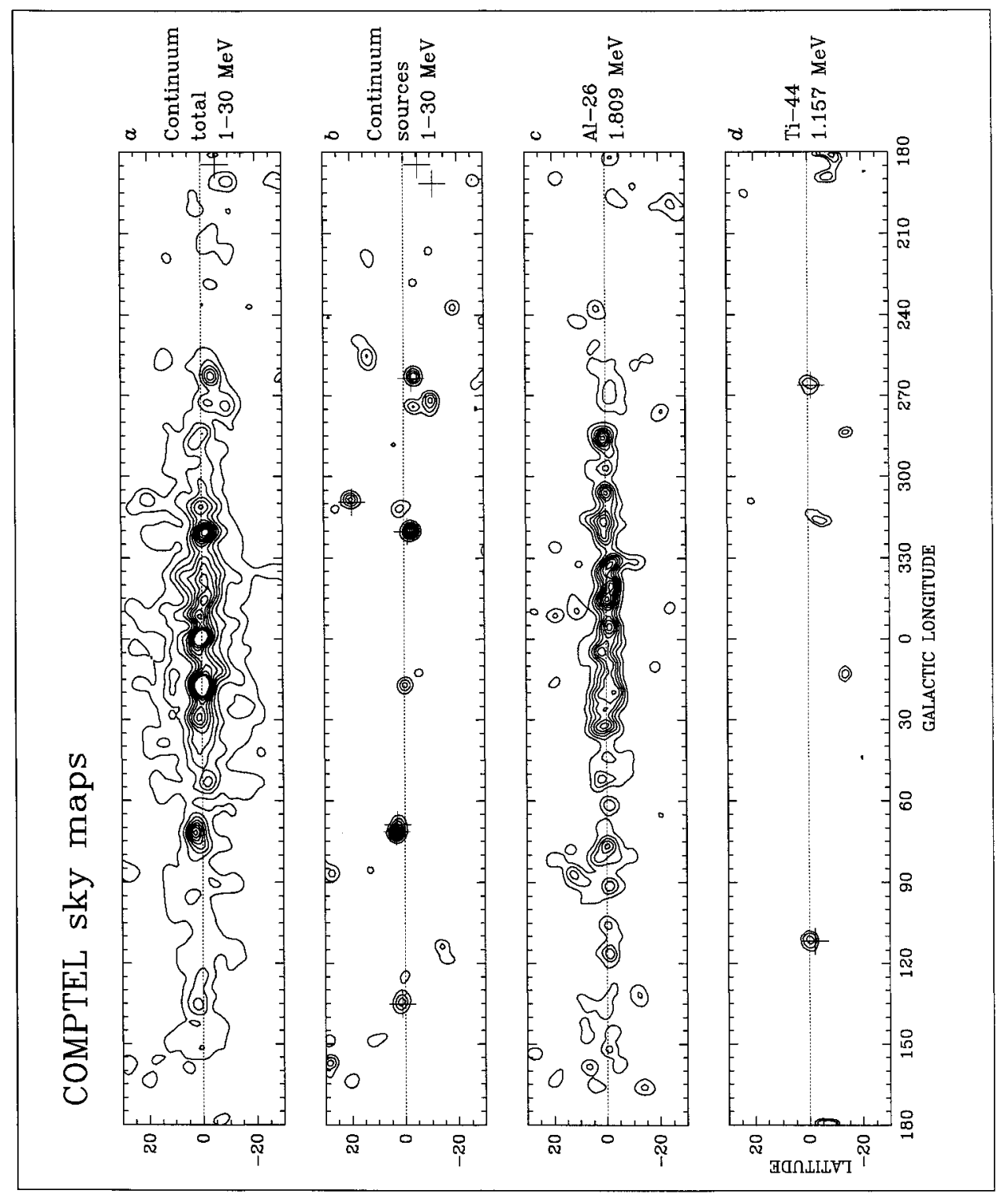

FIGURE 1. Examples of new COMPTEL maps of the Milky Way. Panels abe include data obtained during the first $\sim 5$ years of the mission (VPs 1-522.5); panel $d$ includes data up to VP 617.1. The Crab is modeled out in the two continuum maps. Clearly visible in panel $b$ are e.g. the Vela pulsar (263,-3), PSR 1509-58 (320,-1), Cyg X-1 (71,3), 2CG135+1 (135,1), Cen A $(310,19)$, a strong unidentified source at $(18,0)$, and a peculiar extended source centered at about $(272,-8)$ [16]. In panel $d$ the locations of Cas $\mathrm{A}$ and the new candidate ${ }^{44} \mathrm{Ti}$ source $[11,12]$ are indicated. 




FIGURE 2. COMPTEL spectrum of the inner Galaxy (statistical errors and $25 \%$ errors, including systematics), together with results from Ginga [29], OSSE [19,20], and EGRET [24].

to be judged from dedicated studies (see [15] for a general discussion on maximumentropy mapping of COMPTEL data). Figs. 2 shows the resulting spectrum for the inner galactic radian. Spectra for the individual model components are available as well [4]. These can be compared with e.g. bremsstrahlung and IC model spectra, but this is beyond the scope of this paper. Also a spectrum of the isotropic (cosmic) background emission is extracted in this analysis (see [6]), which turns out to be in good agreement with the result of a very different analysis technique $[13,28]$.

\section{LINE EMISSION}

The background that needs to be accounted for in $\gamma$-ray line studies (main focus here is on the ${ }^{26} \mathrm{Al} 1.809 \mathrm{MeV}$ line) consists of an instrumental component and the celestial continuum emission. We have previously treated these two components as one entity, both in our sky mapping $[15,17,18]$ and in our sky-model fitting $[8,9,14]$. We determined in those studies the background model at $1.7-1.9 \mathrm{MeV}$ (hereafter referred to as the $1.8 \mathrm{MeV}$ band) by scaling of the total event distribution at adjacent energies $(\sim 1-10 \mathrm{MeV}$, excluding the $1.8 \mathrm{MeV}$ band). This is an approximation, because the spectra of the instrumental and celestial components are different. As the instrumental component strongly dominates, the celestial continuum emission is not properly accounted for. We have now applied the same method as in our continuum study, but in a two-step approach, first extracting the celestial emission in narrow energy ranges adjacent to the $1.8 \mathrm{MeV}$ band and then using the derived (interpolated) intensity distribution as a fixed ingredient in the analysis of the $1.8 \mathrm{MeV}$ band itself. Further details can be found in [5].

Fig. 1c shows the resulting $1.809 \mathrm{MeV}$ maximum-entropy map. The appearance of this map is similar to that of the map obtained with our previous background modeling method [17] (an update is presented in these proceedings [18]). In the present new analysis, however, we tend to extract a higher flux for the galactic disk 
at large (e.g $\sim 4 \times 10^{-4} \gamma \mathrm{cm}^{-2} \mathrm{~s}^{-1} \operatorname{rad}^{-1}$ for the inner galactic radian, $|b|<20^{\circ}$ - included in Fig. 2 - which would indeed be in better agreement with results from other experiments). Again, the significance of small-scale map features has to be judged from dedicated studies [15]. Fig. 1d presents a new map in the ${ }^{44} \mathrm{Ti}$ $1.157 \mathrm{MeV}$ line. More detailed results from this analysis for Cas $\mathrm{A}$ and the new candidate ${ }^{44} \mathrm{Ti}$ source are discussed elsewhere in these proceedings [12].

In conclusion: We seem to have gradually learned how to account accurately for the time-variable intense instrumental background of COMPTEL, despite the fact that an independent estimate of it in the 3-dimensional data space is lacking. A particularly nice aspect of the developed analysis technique is that it can be applied to essentially all scientific topics covered by COMPTEL (sources, extended emission, lines) providing images as well as spectral information.

ACKNOWLEDGMENTS: The COMPTEL project is supported by the German government through DARA grant 50 QV 90968, by NASA under contract NAS5-26645, and by the Netherlands Organisation for Scientific Research (NWO).

\section{REFERENCES}

1. Bloemen H., et al., in Compton Gamma-Ray Obs., eds. M. Friedlander et al., p. 30 (1993).

2. Bloemen H., et al., Ap.JS 92, 419 (1994).

3. Bloemen H., et al., Proc. 4th Compton Symp., eds. C.D. Dermer et al., p. 1074 (1997).

4. Bloemen H., et al., Proc. 3rd Integral Symp., in press [sky mapping] (1999a).

5. Bloemen H., et al., Proc. 3rd Integral Symp., in press [26 Al study] (1999b).

6. Bloemen H., et al., Proc. 3rd Integral Symp., in press [cosmic background] (1999c).

7. Collmar W., et al, these proceedings

8. Diehl R., et al., $A \& A$ 298, 445 (1995).

9. Diehl R., et al., Proc. 4th Compton Symp., eds. C.D. Dermer et al., p. 1114 (1997).

10. van Dijk, R., Ph. D. thesis, University of Amsterdam (1996).

11. Iyudin, A., et al., Nature 396, 142 (1998).

12. Iyudin, A., et al., these proceedings

13. Kappadath S.C., Ph. D. thesis, Univ. of New Hampshire (1998).

14. Knödlseder J., et al., $A \& A, \mathbf{3 4 4}, 68$ (1999a).

15. Knödlseder J., et al., $A \& A, \mathbf{3 4 5}, 813$ (1999b).

16. van der Meulen R.D., et al., Proc. 3rd Integral Symp., in press (1999).

17. Oberlack U., et al., A $8 A S$ 120, C311 (1996).

18. Plüschke S., et al., these proceedings

19. Purcell W.R., et al., Proc. 24th Int. Cosmic Ray Conf. 2, 211 (1995).

20. Purcell W.R., et al., AEAS 120, C389 (1996).

21. Schönfelder V., et al., ApJS 86, 657 (1993).

22. Strong A.W., et al., $A \& A$ 292, 82 (1994).

23. Strong A.W., et al., A $B A S$ 120, C381 (1996).

24. Strong A.W. \& Mattox J., A $8 A$ 308, L21 (1996).

25. Strong A.W., et al., in The Transparent Universe, eds. Winkler et al., p. 533 (1997a).

26. Strong A.W., et al., Proc. 4th Compton Symp., eds. C.D. Dermer et al., p. 1198 (1997b).

27. Strong A.W., Moskalenko I. \& Schönfelder V., Proc. 25th ICRC, OG 8.1.1 (1997c).

28. Weidenspointner G., et al., these proceedings

29. Yamasaki N.Y, et al., A $A A S$ 120, C393 (1996). 